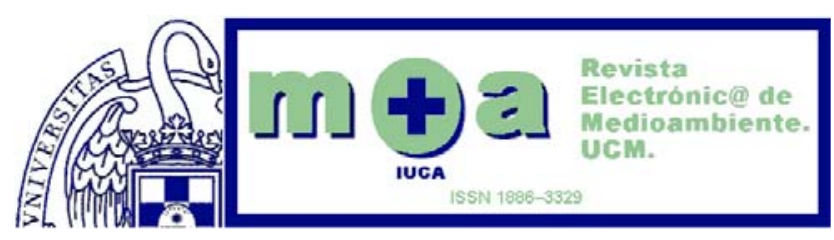

\title{
Estudio de la Evolución de la Gestión Ambiental en Empresas Industriales
}

\author{
Marta Ormazabal \\ Departamento de Organización Industrial. Tecnun, Universidad de Navarra. \\ mogoenaga@tecnun.es \\ J ose María Sarriegi \\ Departamento de Organización Industrial. Tecnun, Universidad de Navarra. \\ imsarriegi@tecnun.es
}

Recibido: 11 de noviembre de 2011

Aceptado: 5 de diciembre de 2011

\section{RESUMEN}

La gestión ambiental se ha convertido en un aspecto esencial en todas las empresas. Sin embargo, no todas han alcanzado el mismo nivel de madurez. A través de una serie de workshops con expertos en el campo ambiental, se ha recogido información referente a aquellos aspectos que afectan a la gestión ambiental de todas las empresas para lograr demostrar que la evolución de la gestión ambiental a lo largo del tiempo es similar en todas ellas. Se ha definido una serie de factores, políticas, indicadores y comportamientos que intervienen en la gestión ambiental a lo largo del tiempo. Este estudio permite a las empresas identificar en qué estado de madurez se encuentran y qué pasos tienen que ir dando hacia la excelencia ambiental.

Palabras claves: gestión ambiental, madurez, fase

\section{Study of the Evolution of Environmental Management in Industrial Enterprises}

\begin{abstract}
Environmental Management has recently become an essential issue for companies. Nevertheless, not all the companies have reached the same maturity level. Through some workshops with environmental experts, information on those aspects that affect environmental management has been collected to demonstrate that the evolution of environmental management is similar in every company. Some factors, policies, indicators and behaviors that affect environmental management over time have been defined. This study allows companies to identify in which maturity state they are in and what are the steps that they have to follow to go towards environmental excellence.
\end{abstract}

Key Words: environmental management, maturity, phase 


\section{Étude de I'évolution de la Gestion de I'Environnement dans les Entreprises Industrielles}

\section{RÉSUMÉ}

Actuellement, la gestion de l'environnement est devenue essentielle dans toutes les entreprises; mais pas toutes les entreprises ont atteint le même niveau de maturité. Grâce à plusieurs ateliers menés avec des experts dans le domaine de I' environnement, il y a des informations sur les aspects qui touchent la gestion de l'environnement de toutes les entreprises afin de démontrer que, au fil du temps, la gestion de I'environnement va évoluer de la même manière dans toutes les entreprises. Cette étude a identifié une série de facteurs, politiques, indicateurs et comportements impliqués dans la gestion de l'environnement au fils du temps, permettant aux entreprises $d$ 'identifier l'état de maturité où ils sont et let étapes qu'ils doivent suivre pour atteindre I'excellence environnementale.

Mots-clés: gestion de l'environnement, maturité, phase.

\section{I NTRODUCCI ÓN}

Las empresas cada vez son más conscientes de que la gestión ambiental es un factor que deben tener en cuenta en el día a día de sus actividades. El consumo excesivo de los recursos naturales, así como el aumento de la generación de residuos están derivando en una mayor exigencia a las empresas por parte de la legislación y de la sociedad (Claver et al. 2007, Zorpas 2010). Los clientes, como parte de la sociedad, exigen ya mayores medidas ambientales, así como procesos, productos y servicios más respetuosos con el medio ambiente. Por tal causa, se está produciendo un aumento en la demanda de productos y servicios que minimicen el impacto ambiental (Abarca and Sepúlveda 2001), así como unos procesos más limpios (Vachon and Klassen 2008).

La Gestión Ambiental de una empresa tiene como objetivo principal reducir el impacto ambiental generado por la misma (Cramer 1998, Céspedes-Lorente et al. 2003). Esto incluye el análisis de todo el ciclo de vida del producto o servicio; es decir, diseñar los productos y/o servicios desde la obtención de materia prima hasta el final de la vida útil del producto intentando reducir al máximo los impactos ambientales (Ramirez and Galán 2006, Aoe 2007). De esta forma, utilizando herramientas ligadas al eco-diseño se pueden llegar a crear nuevos productos, procesos y servicios que disminuyen el impacto ambiental.

Los aspectos ambientales no deben ser responsabilidad únicamente del departamento de medio ambiente, sino que deben ser abordados por todas las áreas de la empresa, incluyendo a los proveedores (Chiappeta and Almada 2008). En la literatura se observa que para alcanzar la excelencia ambiental tiene que existir compromiso por parte de la Dirección, unos recursos adecuados para poder implementar las medidas ambientales necesarias, comunicación y entrenamiento de los trabajadores de la empresa, y auditorías integradas en el sistema para poder hacer un seguimiento de los aspectos ambientales (Zutshi and Sohal 2005, Tarí et al. 2010).

Los sistemas de gestión y las certificaciones se han convertido en una parte imprescindible en la organización y en un requisito para la supervivencia en el siglo XXI (Zutshi and Sohal 2005). Durante años, las consultoras ambientales han ofrecido a las empresas ayuda en la implementación de distintas certificaciones para mejorar la gestión ambiental de las mismas. Algunas de estas certificaciones son la serie de normas ISO 14000 a nivel internacional y el EMAS a nivel europeo.

No todas las empresas han alcanzado el mismo nivel de madurez de gestión ambiental. Algunas empresas contemplan la gestión ambiental como un instrumento para mejorar la eficiencia de sus procesos, al mismo tiempo que cuidan el medio ambiente (Lozano and Vallés 2007). Muchas otras empresas se limitan a cumplir los requisitos de la legislación 
y de los clientes, pero no ven más allá, ni conocen los pasos que deben dar para seguir mejorando en temas ambientales.

A través de este trabajo de investigación se pretende demostrar que la evolución de la gestión ambiental es similar en todas las empresas industriales. Para ello, este artículo se centra en identificar aquellas variables que intervienen en todo Sistema de Gestión Ambiental (SGA) y permiten explicar su evolución. A través de workshops con expertos en la gestión ambiental de empresas industriales, se recopiló información acerca de la evolución de la GA en varias empresas.

Gracias a esta investigación las empresas van a poder comprender mejor la evolución de la gestión ambiental y van a poder avanzar hacia la excelencia ambiental; ya que este estudio les va a permitir conocer en qué estado de madurez se encuentran y cuáles son los pasos que deben dar para seguir mejorando.

\section{METODOLOGÍA DE INVESTIGACIÓN. GROUP MODEL BUI LDI NG ( GMB)}

Group Model Building es una metodología colaborativa que permite capturar información de un grupo de personas que son expertas en el problema a tratar. Para ello es necesario identificar y disponer de expertos pueden aportar su conocimiento desde distintos puntos de vista. En este caso se seleccionaron personas expertas en la gestión ambiental de distintas empresas, consultorías ambientales y académicos con experiencia en proyectos ambientales. Para recopilar toda esta información se realizaron una serie de workshops en donde se reunieron los expertos.

La metodología GMB define una serie de roles para que los workshops sean lo más efectivos posibles. Estos roles son el facilitador, el modelador, el 'process coach', el registrador y el 'gatekeeper' (Richardson and Andersen 1995).

A la hora de recoger la información planteada por los expertos, la metodología GMB utiliza diferentes ejercicios. En el primer ejercicio, se identifican los agentes que intervienen en la gestión ambiental y se clasifican según el interés y la influencia en el problema investigado. En el segundo, se identifican las políticas que pueden afectar a la evolución de la gestión ambiental. En el tercero, se establecen los indicadores asociados a las distintas políticas identificadas y los resultados que pueden obtenerse. En el último ejercicio, los grupos de expertos elaboran las gráficas asociadas a los indicadores sobre las posibles evoluciones que pueden seguir los mismos según va madurando la gestión ambiental de la empresa.

\section{IDENTI FI CACIÓN DE LOS STAKEHOLDERS}

Los stakeholders identificados por los expertos son los representados en la

Figura 1. Todos ellos se han ordenado según su interés y su influencia en la Gestión Ambiental de una empresa. 


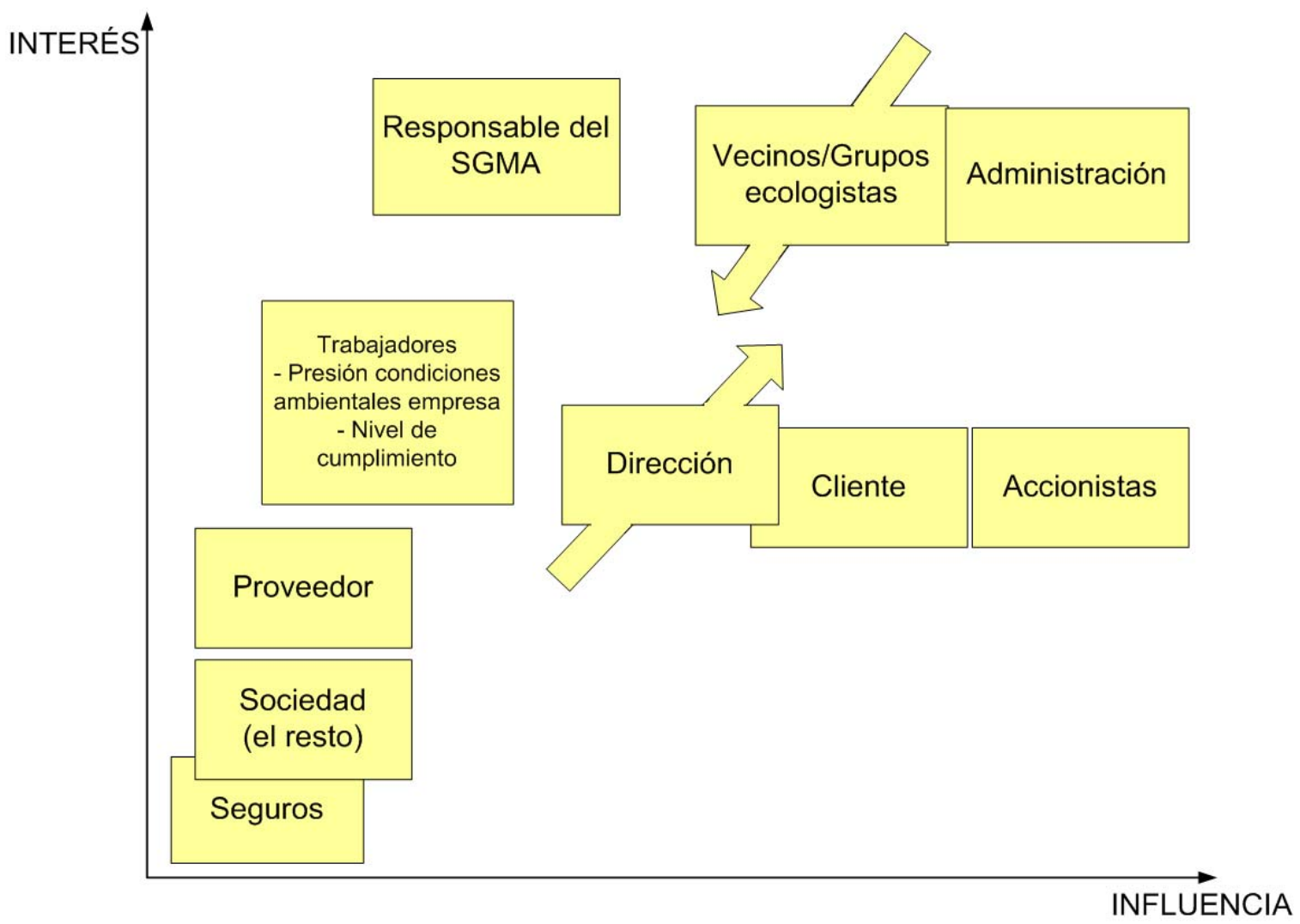

Figura 1. I dentificación de stakeholders

Las compañías de seguros de una empresa pueden obligar a cumplir una serie de requisitos ambientales. Sin embargo, su interés en aspectos ambientales es todavía reducido y su influencia es muy pequeña. Otro stakeholder identificado es la Sociedad. En este stakeholder no se incluye a los clientes. Por este motivo, el interés y la influencia que pueden presentar en la gestión ambiental de una empresa particular es bastante bajo. Los proveedores de las empresas tienen un interés bajo-medio y una influencia bastante limitada. Los trabajadores tienen un interés medio en la gestión ambiental de la empresa y su influencia es bastante baja ya que no intervienen en las decisiones sobre esta gestión. El Responsable del Sistema de Gestión Medio Ambiental tiene mucho interés en disponer de una gestión ambiental excelente, pero su influencia no es muy grande. La Dirección no presenta demasiado interés y su influencia es media. Sin embargo, según va madurando la gestión ambiental de la empresa, la Dirección también tendrá más interés e influencia

). Los clientes tienen un interés medio, pero que va creciendo con el tiempo, y la influencia es bastante grande, ya que la empresa debe responder a sus requisitos. Los accionistas tienen un interés medio, pero la influencia es muy grande, ya que son los que aportan el capital de la empresa.

Como se puede observar, aquellos que más interés y más influencia tienen en la gestión ambiental de una empresa son los Vecinos/Grupos ecologistas y la Administración. Los Vecinos tienen mucho interés, ya que los impactos ambientales de la empresa pueden afectarles directamente a ellos y tienen bastante influencia, ya que pueden presionar significativamente para que se consigan ciertos logros ambientales. Y los Grupos ecologistas tienen bastante interés en que cada empresa tenga menos impactos ambientales y bastante influencia. Esta influencia e interés de los vecinos y de los Grupos ecologistas va decreciendo con el tiempo a medida que la gestión ambiental de la empresa mejora. La Administración tiene un interés muy alto y es la que más influencia 
tiene, ya que las empresas tienen que cumplir con la legislación que se les impone, sino serán penalizadas.

\section{I DENTI FI CACIÓN DE LAS POLÍ TI CAS}

En este ejercicio los expertos han identificado aquellas políticas que se pueden implementar para mejorar la gestión ambiental. Una vez identificadas las distintas

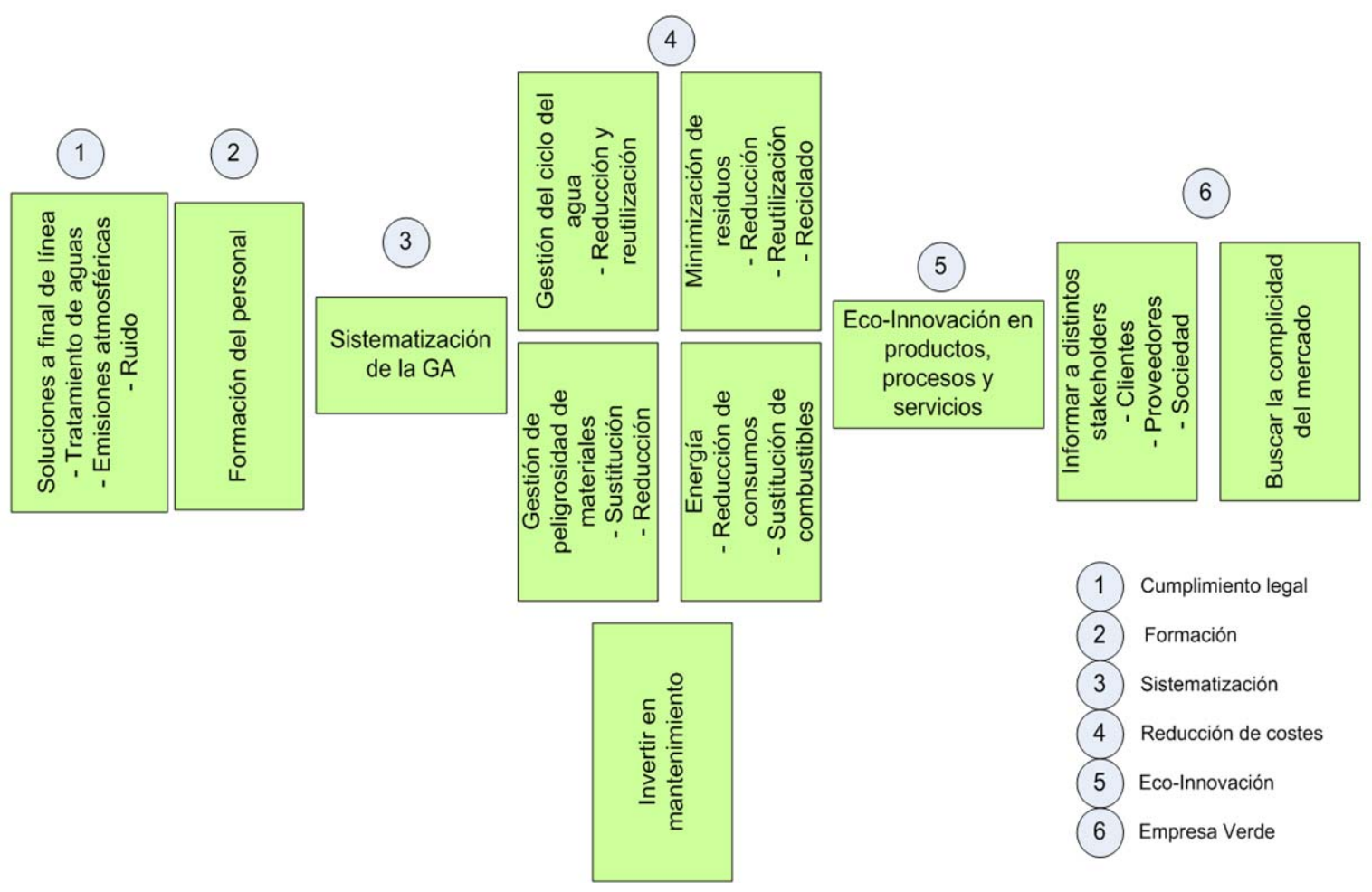

políticas, se han agrupado en 6 conjuntos, que posteriormente se han ordenado de forma secuencial, según el orden temporal en el que se implementan en las empresas

). Estos seis grupos son Cumplimiento legal, Sistematización, Certificación, Reducción de costes, Proactividad y Empresa Verde.

El primer grupo y, por lo tanto, la primera fase, es el Cumplimiento legal. Cualquier empresa, el primer paso que da en cuestiones ambientales, es debido a que la ley se lo exige. En esta etapa las políticas que se implementan son las soluciones a final de línea, que son aquellas soluciones técnicas que se implementan al final del proceso productivo. Algunas de las soluciones pueden ser el tratamiento de aguas, el equipamiento para reducir las emisiones atmosféricas, así como la disminución del ruido.

La segunda fase es la Formación del personal. Se imparte formación a todos los trabajadores para que sean capaces de utilizar eficazmente los equipamientos con los que cuenta la empresa y para poder implementar satisfactoriamente las medidas ambientales que va tomando la empresa.

La tercera fase es la Sistematización de la gestión ambiental. Una vez tomadas ciertas medidas, se comienza a formalizar el sistema y, en ocasiones, a certificarse, de tal forma 
que la empresa tenga un reconocimiento externo del SGA. El objetivo es tener identificados y monitorizados todos los aspectos medioambientalmente relevantes de la empresa.

En la cuarta fase, las empresas se orientan hacia la Reducción de costes. Para conseguir esta minimización de costes, algunas de las políticas que se implementan son la gestión del ciclo del agua, de los materiales peligrosos, y de la energía. También es necesaria la inversión en mantenimiento para conseguir la máxima utilidad de las máquinas.

La quinta fase refleja la eco-innovación de la empresa. En esta fase se utilizan herramientas ambientales para innovar en productos, procesos y/o servicios y poder así diferenciarse de la competencia. Entre estas herramientas se encuentra el Ecodiseño y Análisis del Ciclo de Vida (ACV), la huella de carbono y el etiquetado ecológico.

Y por último, la sexta fase se ha denominado Empresa Verde. Durante esta fase, se informa a los distintos stakeholders involucrados en la gestión ambiental de la empresa de las medidas y progresos que van teniendo lugar en la empresa para que se empiece a conocer a la empresa como verde. Por ese motivo, la imagen verde de la empresa comienza a desarrollarse y el mercado aprecia las medidas ambientales de la empresa. EI objetivo de esta fase es capitalizar los esfuerzos realizados hasta el momento.

Figura 2. Identificación de políticas

\section{I NDI CADORES DE LAS POLÍTI CAS}

Para cada una de estas seis políticas se han identificado una serie de indicadores que se han agrupado en inductores, que son aquellos indicadores que reflejan la cantidad de esfuerzo que hace falta para que la política se lleve a cabo; y resultados, aquellos que estiman las consecuencias de la implantación de las políticas (Tabla 1).

\begin{tabular}{|c|c|c|c|c|c|c|}
\hline $\begin{array}{l}\text { Política } \\
\text { s }\end{array}$ & 1 & 2 & 3 & 4 & 5 & 6 \\
\hline $\begin{array}{l}\text { I } \\
\mathbf{N} \\
\mathbf{D} \\
\mathbf{U} \\
\mathbf{C} \\
\mathbf{T} \\
\mathbf{O} \\
\mathrm{R} \\
\mathrm{E} \\
\mathbf{S}\end{array}$ & $\begin{array}{l}\text { - Inversión } \\
\text { en } \\
\text { actuaciones } \\
\text { a final de } \\
\text { línea(Euros) } \\
\text {-Exigencias } \\
\text { ambientales }\end{array}$ & $\begin{array}{l}\text { - Horas } \\
\text { impartidas } \\
\text { de formación }\end{array}$ & $\begin{array}{l}\text { - Horas } \\
\text { trabajo } \\
\text { dedicadas a } \\
\text { la gestión } \\
\text { ambiental }\end{array}$ & $\begin{array}{l}\text { - } \\
\text { Inversione } \\
\text { s en MTD }\end{array}$ & $\begin{array}{l}\text { - } \\
\text { Herramienta } \\
\text { s } \\
\text { ambientales } \\
\text { (etiquetas, } \\
\text { ACV, CO2...) }\end{array}$ & $\begin{array}{l}\text {-Comunicación } \\
\text { externa }\end{array}$ \\
\hline $\begin{array}{l}\text { R } \\
\text { E } \\
\text { S } \\
\text { U } \\
\text { L } \\
\text { T } \\
\text { A } \\
\text { D } \\
\text { O } \\
\text { S }\end{array}$ & $\begin{array}{l}\text {-Impacto } \\
\text { ambiental } \\
- \\
\text { Cumplimient } \\
\text { o exigencias }\end{array}$ & $\begin{array}{l}\text { - } \\
\text { Cumplimient } \\
\text { o exigencias }\end{array}$ & $\begin{array}{l}\text { - Estado } \\
\text { certificacione } \\
\text { s }\end{array}$ & $\begin{array}{l}\text {-Consumos } \\
\text {-I mpacto } \\
\text { ambiental }\end{array}$ & $\begin{array}{l}\text { - Nuevos } \\
\text { productos, } \\
\text { procesos, } \\
\text { servicios } \\
\text { verdes }\end{array}$ & $\begin{array}{l}\text { - Impactos } \\
\text { Prensa } \\
- \\
\text { Reconocimient } \\
\text { o exterior } \\
\text { (premios...) }\end{array}$ \\
\hline
\end{tabular}

Tabla 1. Identificación de indicadores

En la primera fase de Cumplimiento legal, los indicadores definidos como inductores son la Inversión en actuaciones a final de línea; es decir, los euros invertidos para actuaciones medioambientales a final de línea; y las exigencias ambientales requeridas en la legislación. Como resultado, se mide el impacto ambiental producido y el cumplimiento de las exigencias. 
En la segunda fase de Formación se miden las horas impartidas. Como resultado de esta fase, el indicador propuesto es el cumplimiento de las exigencias ambientales.

En la tercera fase de Certificación ambiental, se miden las horas de trabajo dedicadas a la gestión ambiental y como resultado el estado de las certificaciones; es decir, comprobar si se van consiguiendo certificaciones que requieren mejoras ambientales mayores.

La cuarta fase se focaliza en la reducción de costes. En esta fase el indicador utilizado es la inversión realizada en las Mejores Tecnologías Disponibles (MTD). Como resultado se mide su efecto en los consumos, y el impacto ambiental para comprobar que realmente mejora.

La quinta fase se centra en la Eco-Innovación. Los indicadores inductores son las herramientas ambientales utilizadas como el etiquetado ecológico, el ecodiseño y el análisis del ciclo de vida. Como resultado se miden los nuevos productos, procesos y/o servicios verdes propuestos.

En la última fase de Empresa Verde, se mide la comunicación externa a los diferentes stakeholders sobre las distintas actuaciones ambientales llevadas a cabo en la empresa. Como resultado se miden los impactos generados en la prensa y los reconocimientos asignados a la empresa por su excelencia ambiental.

\section{COMPORTAMI ENTO EN EL TIEMPO}

En este ejercicio, los expertos tratan de graficar en el tiempo algunos de estos indicadores, para ver su evolución (Figura 3). Algunos indicadores no se han graficado ya que no aportan valor extra al gráfico. Es el caso, por ejemplo, de los consumos, ya que están muy relacionados con las inversiones que se hagan de MTD para intentar reducirlos. Tampoco se grafican los nuevos productos, procesos y/o servicios verdes, ya que son directamente proporcionales a las herramientas ambientales utilizadas en la empresa. Por último los impactos en la prensa y el reconocimiento exterior van ligados implícitamente a la comunicación exterior.

Esta gráfica no es absolutamente precisa, sino que intenta representar el comportamiento de cada uno de estos indicadores a lo largo del tiempo. Las seis fases corresponden a las anteriormente identificadas.

Como se ha comentado previamente, la primera fase corresponde al cumplimiento legal, cuando la principal causa de mejora ambiental es la legislación. Esta fase puede tener una duración aproximada de 24 meses. Existe una inversión muy grande al final de las líneas de producción para tratar de cumplir las exigencias ambientales, que se mantienen estables en esta fase. Inicialmente existen muchos impactos, pero van disminuyendo a medida que se invierte. También se observa que el cumplimiento de las exigencias va creciendo y se empiezan a impartir algunas horas de formación.

La siguiente fase dura aproximadamente 3 meses. Las exigencias medioambientales crecen un poco, las inversiones a final de línea decrecen, ya que la inversión más grande ya se ha realizado y el cumplimiento de las exigencias va creciendo y se va aproximando a las exigencias ambientales. Por este motivo, los impactos van disminuyendo. Existen muchas horas de formación al personal. En esta fase, comienzan a crecer las horas dedicadas a la formalización de la gestión ambiental.

En las siguientes fases, las exigencias ambientales van creciendo poco a poco y el cumplimiento de las exigencias va creciendo. Este cumplimiento se debe a la puesta en marcha de mejoras ambientales, que al mismo tiempo hacen que los impactos vayan decreciendo considerablemente a lo largo del tiempo. Las inversiones a final de línea se convierten en mantenimiento y van decreciendo poco a poco. Las horas de formación van decreciendo poco a poco, aunque existen picos de formación cuando se introducen 
nuevas medidas ambientales. A partir de la tercera fase, empiezan a existir más horas de trabajo dedicadas a Gestión Ambiental, aunque también se refleja que existen picos a lo largo de la evolución de un Sistema de Gestión Ambiental debido a la aparición de medidas ambientales concretas. A partir de la fase 4, la empresa se certifica y ese reconocimiento externo da lugar en la sexta fase a herramientas más avanzadas como el análisis del ciclo de vida. En la fase 4 también empiezan las inversiones para las Mejores Tecnologías Disponibles (MTD). Estas inversiones se mantienen estables a lo largo del tiempo, aunque tienen algunas subidas en momentos en los que se hacen grandes inversiones para adquirir nuevas tecnologías. Por otro lado, a partir de la quinta fase, se dedican horas de trabajo para fomentar la participación, ya sea a través de concursos, sugerencias o cualquier otro método participativo. A partir de la sexta fase comienza la comunicación externa, donde se hacen públicas las mejoras ambientales de la empresa y los clientes y la Sociedad empiezan a reconocer a la compañía como empresa verde.

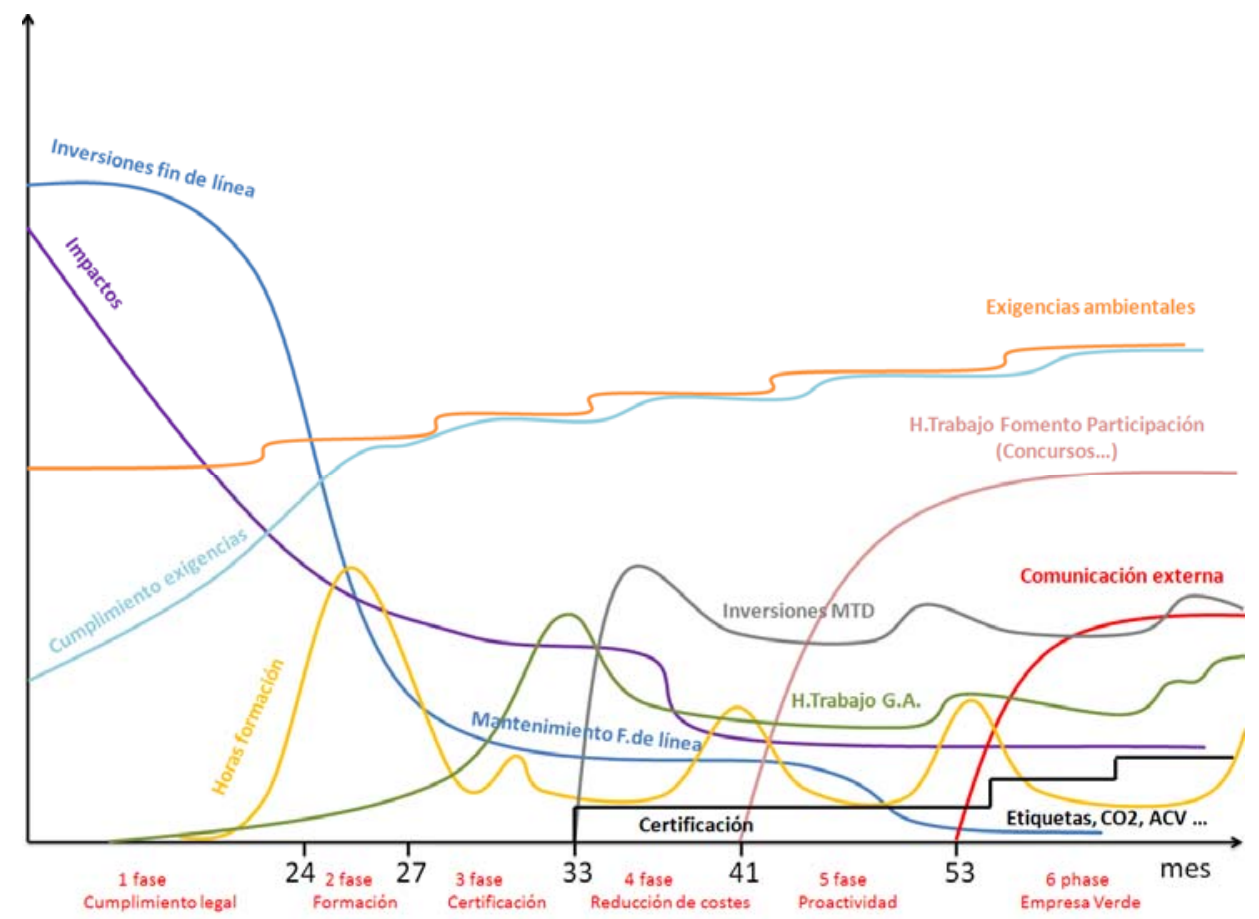

Figura 3. Comportamiento de los indicadores

\section{CONCLUSI ONES}

Este trabajo de investigación ha identificado los agentes, políticas e indicadores que definen la evolución de un Sistema de Gestión Ambiental, de tal forma que las empresas puedan entender cómo funciona y sean capaces de identificar los pasos que deben dar para alcanzar la excelencia medioambiental. Como se ha comprobado la Gestión Medioambiental de muchas empresas evoluciona de manera similar y este artículo describe en detalle ese patrón de comportamiento. Aunque las empresas presentan diferencias significativas cuando se las observa en detalle, este trabajo de investigación ha demostrado que observando su evolución de manera agregada, se pueden observar similitudes significativas.

\section{BI BLI OGRAFÍ A}


Abarca R, Sepúlveda S (2001) Eco-etiquetado: Un Instrumento para Diferenciar Productos e Incentivar la Competitividad. Comercio-Ambiente, vol. 17, pp. 1-65.

Aoe T (2007) Eco-efficiency and ecodesign in electrical and electronic products. Journal of Cleaner Production, vol. 15, no. 15, pp. 1406-1414.

Céspedes-Lorente J, Burgos-Jiménez Jd \& Álvarez-Gil MJ (2003) Stakeholders' environmental influence. An empirical analysis in the Spanish hotel industry. Scandinavian Journal of Management, vol. 19, no. 3, pp. 333-358.

Chiappeta CJ, Almada FC (2008) Relationships between human resource dimensions and environmental management in companies: proposal of a model. Journal of Cleaner Production, vol. 16.

Claver E, López MD, Molina JF \& Tarí JJ (2007) Environmental management and firm performance: A case study. J ournal of Environmental Management, vol. 84.

Cramer J (1998) Environmental Management: From 'fit' to 'stretch'. Business Strategy and the Environment, vol. 7, no. 3, pp. 162-172.

Lozano M, Vallés J (2007) An analysis of the implementation of an environmental management system in a local public administration. Journal of Environmental Management, vol. 82, pp. 495-511.

Ramirez E, Galán L (2006) El Ecodiseño Como Herramienta Básica de Gestión Industrial. , pp. 32.

Richardson GP, Andersen DF (1995) Teamwork in Group Model Building. System Dynamics Review, vol. 11, no. 2, pp. 113-137.

Tarí JJ, Claver-Cortés E, Pereira-Moliner J \& Molina-Azorín JF (2010) Levels of quality and environmental management in the hotel industry: Their joint influence on firm performance. International J ournal of Hospitality Management, vol. 29, pp. 500-510.

Vachon S, Klassen RD (2008) Environmental management and manufacturing performance: The role of collaboration in the supply chain. International Journal of Production Economics, vol. 111.

Zorpas A (2010) Environmental management systems as sustainable tools in the way of life for the SMEs and VSMEs. Bioresource technology, vol. 101, no. 6, pp. 1544-1557.

Zutshi A, Sohal AS (2005) Integrated management system. The experiences of three Australian organisations. Journal of Manufacturing Technology Management, vol. 16, no. 2, pp. 211-232.

\section{RESEÑAS BIOGRÁFI CAS}

\section{Marta Ormazabal}

Marta Ormazabal Goenaga (San Sebastián, 1986) es Ingeniera Industrial por TECNUN, Escuela Superior de Ingenieros de San Sebastián de la Universidad de Navarra (2009). Actualmente está realizando su tesis doctoral en el departamento de Organización Industrial de TECNUN y su área de investigación está centrada en la Gestión Ambiental. Así mismo, durante el curso académico 2010-2011 fue ayudante en la asignatura "Administración de Empresas" y actualmente es ayudante de la asignatura "Modelización y Simulación". Entre sus últimas publicaciones se encuentran "Environmental Management in Industrial Enterprises" y "Environmental management model and definition of the different maturity states", por la cual obtuvo el premio de presentación oral en el congreso internacional QMOD 2011.

\section{J ose María Sarriegi Dominguez}

Jose Mari Sarriegi (Beasain, 1970) es Ingeniero Industrial y Doctor por la Universidad de Navarra. Actualmente, es profesor de Sistemas de Información, Gestión del Conocimiento y Modelización y Simulación del Departamento de Organización Industrial de TECNUN, el campus tecnológico de Universidad de Navarra en San Sebastián. Su línea de investigación se centra en la modelización de sistemas complejos, tales como la gestión 
de la seguridad de los sistemas de información, la gestión de grandes crisis y la gestión ambiental. Junto con Marta Ormazábal ha publicado entre otros artículos "Environmental Management in Industrial Enterprises" y "Environmental management model and definition of the different maturity states". Asimismo, tiene publicaciones en otros campos, todos ellos unidos por la modelización. 